PROCEEDINGS OF THE AMERICAN MATHEMATICAL SOCIETY

Volume 125, Number 3, March 1997, Pages 635-647

S 0002-9939(97)03529-6

\title{
BOUNDARY VALUES OF HOLOMORPHIC SEMIGROUPS
}

\author{
WOLFGANG ARENDT, OMAR EL MENNAOUI, AND MATTHIAS HIEBER \\ (Communicated by Palle E. T. Jorgensen) \\ Dedicated to Professor H. H. Schaefer on the Occasion of his 70th Birthday
}

\begin{abstract}
The concept of boundary values of holomorphic semigroups is used to give a new proof of a result due to Hörmander, saying that the operator $i \Delta$ generates a $C_{0}$-semigroup on $L^{p}\left(\mathbb{R}^{N}\right)$ if and only if $p=2$. Using a recent result on Laplace transforms by Prüss one obtains by this theory also a new proof of the classical characterization theorem of holomorphic semigroups.
\end{abstract}

\section{INTRODUCTION}

The starting point of the present paper is a classical result of Hörmander [Hö] saying that the operator $i \Delta$ generates a $C_{0}$-semigroup on $L^{p}\left(\mathbb{R}^{N}\right)$ if and only if $p=2$. Hörmander's proof is based on euclidean harmonic analysis. In fact, he shows that for each $t>0$, the function $\xi \mapsto e^{i t|\xi|^{2}}$ (the symbol of $e^{i t \Delta}$ ) is not a multiplier for $L^{p}\left(\mathbb{R}^{N}\right)$ if $p \neq 2$.

There is a completely different way to look at his result. Indeed, observe that the Laplacian $\Delta$ generates a holomorphic $C_{0}$-semigroup of angle $\pi / 2$ on $L^{p}\left(\mathbb{R}^{N}\right)$, $1 \leq p<\infty$. We use the following simple result on the boundary of a holomorphic semigroup (see Section 1 for the proof) to prove Hörmander's result in an elementary way.

Proposition 0.1. Let $A$ be the generator of a holomorphic $C_{0}$-semigroup $T$ on some Banach space $E$ of angle $\pi / 2$. Then iA generates a $C_{0}$-semigroup on $E$ if and only if $T$ is bounded on $\Omega:=\{z \in \mathbb{C} ; \operatorname{Re} z>0, \operatorname{Im} z \geq 0,|z| \leq 1\}$.

In fact, the explicit representation of the semigroup $\left(e^{z \Delta}\right)_{\operatorname{Re} z>0}$ as a convolution operator with the Gaussian kernel allows us to show in Section 2 that $e^{z \delta}$ is unbounded on $\Omega$ if $p \neq 2$. In view of Proposition 0.1 we obtain in this way a proof of Hörmander's result.

Another interesting example of a holomorphic semigroup of angle $\pi / 2$ is the Riemann-Liouville semigroup $J$ (describing fractional integration) on $L^{p}(0,1), 1 \leq$ $p<\infty$. It does have a boundary value for $1<p<\infty$. We show this by identifying $J(z)$ with $B^{-z}(\operatorname{Re} z>0)$, where $-B$ generates the translation semigroup on $L^{p}(0,1)$, and by applying the transference principle due to Coifman and Weiss $[\mathrm{C}-\mathrm{W}]$.

In Sections 4 and 5 we consider the inverse problem: Which semigroups are obtained as boundary values of holomorphic semigroups? Given an operator $A$

Received by the editors April 27, 1995 and, in revised form, July 7, 1995.

1991 Mathematics Subject Classification. Primary 47D06, 47F05.

(C)1997 American Mathematical Society 
in $E$ and $\varphi \in(0, \pi / 2)$, we show that the operators $e^{ \pm i \varphi} A$ generate bounded $C_{0^{-}}$ semigroups on $E$ if and only if $A$ generates a bounded holomorphic semigroup of angle $\varphi$. Our proof is based on a Phragmen-Lindelöf type argument and the Hille-Yosida theorem. Using a recent result on Laplace transforms by Prüss [Pr, Proposition 0.1], one obtains by this result also a new proof of the characterization theorem for holomorphic semigroups.

If the angle $\varphi$ equals $\frac{\pi}{2}$, the situation is different; a spectral condition on the generator and a growth condition on the group are needed in addition. More precisely, if $i A$ generates a polynomially bounded group on $E$ and the spectrum of $A$ lies in a left halfplane, then $A$ generates a holomorphic semigroup of angle $\frac{\pi}{2}$. Our proof of this result uses a new result of Phragmen-Lindelöf type allowing the underlying function to be unbounded on a ray. A more complicated and very different proof had been given before in [El2]. The first result in this direction is due to Jazar [Ja1], [Ja2], who considered bounded groups and used spectral calculus for the proof.

\section{THE BOUNDARY OF A HOLOMORPHIC SEMIGROUP}

Let $A$ be an operator in a Banach space $E$. For $\theta \in(0, \pi]$ let

$$
\Sigma_{\theta}:=\{z \in \mathbb{C} ; z \neq 0,|\arg z|<\theta\} .
$$

We say that $A$ generates a bounded holomorphic semigroup of angle $\varphi \in(0, \pi / 2]$, if $A$ generates a $C_{0}$-semigroup $T$ which has a bounded holomorphic extension $\widetilde{T}$ to the sector $\Sigma_{\varphi}$. Denoting the extension $\widetilde{T}$ still by $T$ one has $T\left(z+z^{\prime}\right)=T(z) T\left(z^{\prime}\right)$ for $z, z^{\prime} \in \Sigma_{\varphi}$ and $\lim _{z \rightarrow 0, z \in \Sigma_{\varphi}} T(z)=I$ strongly. Let $A$ be the generator of a holomorphic semigroup $T$ of angle $\varphi \in(0, \pi / 2]$. By this we mean that for all $\theta \in(0, \varphi)$ there exists $\omega \geq 0$ such that $A-\omega$ generates a bounded holomorphic semigroup $T_{\theta}$ of angle $\theta$ (and then $\left.T(z)=e^{\omega z} T_{\theta}(z), z \in \Sigma_{\theta}\right)$. Then, for $\theta \in$ $[0, \varphi),\left(T\left(t e^{i \theta}\right)\right)_{t \geq 0}$ is a $C_{0}$-semigroup and its generator is $e^{i \theta} A$. For more details we refer to $[\mathrm{Da}],[\mathrm{Go}]$ and $[\mathrm{Pa}]$. Throughout this paper we denote by $\sigma(A)$ and $\rho(A)$ the spectrum and the resolvent set of $A$. Furthermore, we put $\Sigma_{\varphi}^{+}:=\Sigma_{\varphi} \cap\{z \in$ $\mathbb{C} ; \operatorname{Im} z \geq 0\}, \Sigma_{\varphi}^{-}:=\Sigma_{\varphi} \cap\{z \in \mathbb{C} ; \operatorname{Im} z \leq 0\}$ and define $D$ by $D:=\left\{z \in \Sigma_{\pi / 2},|z| \leq\right.$ $1\}$. In this first section we are interested in the boundary value of a holomorphic semigroup.

Proposition 1.1. Let $A$ be the generator of a holomorphic $C_{0}$-semigroup $T$ of angle $\varphi \in(0, \pi / 2]$. Assume that

$$
\sup _{z \in \Sigma_{\varphi}^{+} \cap D}\|T(z)\|<\infty .
$$

Then $e^{i \varphi} A$ generates a $C_{0}$-semigroups $T\left(\cdot e^{i \varphi}\right)$ given by

$$
T\left(t e^{i \varphi}\right)=s-\lim _{\varepsilon \rightarrow 0^{+}} T\left(e^{i \varphi} t+\varepsilon\right) .
$$

Proposition 1.1 is an obvious modification of [H-P, Theorem 17.9.1, 17.9.2]. We call the semigroup $T\left(\cdot e^{i \varphi}\right)$ the boundary value of the holomorphic semigroup $T$. Extending $T$ by formula (1.2) one obtains a strongly continuous function $T: \overline{\Sigma_{\varphi}^{+}} \rightarrow$ $\mathcal{L}(E)$ such that

$$
T\left(z+z^{\prime}\right)=T(z) T\left(z^{\prime}\right), \quad z, z^{\prime} \in \overline{\Sigma_{\varphi}^{+}} .
$$


Finally, we say that $T$ has a boundary value provided (1.1) is satisfied. We also remark that the above result can be easily modified to the case where $e^{i \varphi} A$ and $e^{-i \varphi} A$ generate $C_{0}$-semigroups on $E$. Indeed, assuming that

$$
\sup _{z \in \Sigma_{\varphi} \cap D}\|T(z)\|<\infty
$$

it follows that $e^{ \pm i \varphi} A$ generate $C_{0}$-semigroups $T\left(\cdot e^{ \pm i \varphi}\right)$ on $E$ given by $T\left(t e^{ \pm i \varphi}\right)=$ $s-\lim _{\varepsilon \rightarrow 0^{+}} T\left(e^{ \pm i \varphi} t+\varepsilon\right)$. Then $T: \overline{\Sigma_{\varphi}} \rightarrow \mathcal{L}(E)$ is strongly continuous and

$$
T\left(z+z^{\prime}\right)=T(z) T\left(z^{\prime}\right) \quad \text { for } z, z^{\prime} \in \overline{\Sigma_{\varphi}}
$$

In particular, if (1.4) is satisfied for $\varphi=\pi / 2$, then $i A$ generates a $C_{0}$-group.

The following result deals with the converse of Proposition 1.1.

Proposition 1.2. Let $A$ be the generator of a holomorphic $C_{0}$-semigroup $T$ of angle $\varphi \in(0, \pi / 2]$. Assume that $e^{i \varphi} A$ generates a $C_{0}$-semigroup $S$. Then (1.1) is satisfied and thus $S(t)=T\left(t e^{i \varphi}\right)$ for all $t \geq 0$.

Proof. Since the resolvents of $A$ and $e^{i \varphi} A$ commute, it follows that $S(s) T(t)=$ $T(t) S(s)$ for all $s, t \geq 0$. Let $a, b \geq 0$. Then the family $(V(t))_{t \geq 0}$ of operators given by $V(t):=S(b t) T(a t)$ defines a $C_{0}$-semigroup on $E$. Denote its generator by $B$. If $x \in D(A)$, then $\frac{\partial}{\partial t} V(t) x=\left(b e^{i \varphi}+a\right) A V(t) x$. Hence $\left.\frac{\partial}{\partial t} V(t) x\right|_{t=0}=\left(b e^{i \varphi}+a\right) A x$ and therefore $\left(b e^{i \varphi}+a\right) A \subset B$. Consequently, $V(t)=T\left(\left(b e^{i \varphi}+a\right) t\right)$ for all $t \geq 0$. In particular, $S(b) T(a)=V(1)=T\left(b e^{i \varphi}+a\right)$ for all $a, b \geq 0$. Now let $z \in \Sigma_{\varphi}^{+} \cap D$ and write $z=s e^{i \varphi}+t$ for suitable $s, t \geq 0$. Then $T(z)=T(t) S(s)$. Hence there exists a constant $M \geq 0$ such that

$$
\|T(z)\| \leq\|T(t)\|\|S(s)\| \leq M \quad \text { for all } z \in \Sigma_{\varphi}^{+} \cap D .
$$

\section{Boundary values of the Gaussian SEMigroup}

Let $\left(G_{p}(z)\right)_{\operatorname{Re}} z>0$ be the Gaussian semigroup on $L^{p}\left(\mathbb{R}^{N}\right), 1 \leq p<\infty$, i.e. $G_{p}(z)$ is given by $G_{p}(z) f:=k_{z} * f$ for $f \in L^{p}\left(\mathbb{R}^{N}\right)$ and $\operatorname{Re} z>0$, where

$$
k_{z}(x):=\frac{1}{(4 \pi z)^{N / 2}} \exp \left(\frac{-|x|^{2}}{4 z}\right) \quad\left(x \in \mathbb{R}^{N}\right) .
$$

It is well-known that $\left(G_{p}(z)\right)_{\operatorname{Re} z>0}$ is a holomorphic $C_{0}$-semigroup of angle $\pi / 2$ on $L^{p}\left(\mathbb{R}^{N}\right)$ whose generator is the Laplacian $\Delta_{p}$ equipped with maximal domain. Applying Proposition 1.2 to the Laplacian $\Delta_{p}$ we obtain a direct proof of the following result.

Theorem 2.1 (Hörmander [Hö, p. 109]). Let $1 \leq p \leq \infty$. Then the operator $i \Delta_{p}$ generates a $C_{0}$-semigroup on $L^{p}\left(\mathbb{R}^{N}\right)$ only if $p=2$.

Proof. Since $\Delta_{p}^{*}=\Delta_{q}$ for $\frac{1}{p}+\frac{1}{q}=1$, we may restrict ourselves to the case $1 \leq p \leq 2$. Observe first that

$$
\left\|G_{1}(z)\right\|_{\mathcal{L}\left(L^{1}\right)}=\left\|k_{z}\right\|_{L^{1}}=\left(\frac{|z|}{\operatorname{Re} z}\right)^{N / 2} \quad(\operatorname{Re} z>0) .
$$

Since $\Delta_{2}$ is self-adjoint and form negative we have $\left\|G_{2}(z)\right\|_{\mathcal{L}\left(L^{2}\right)}=1$ for all $z \in$ $\{\lambda \in \mathbb{C} ; \operatorname{Re} \lambda>0\}$. Hence, by the Riesz-Thorin interpolation theorem

$$
\left\|G_{p}(z)\right\|_{\mathcal{L}\left(L^{p}\right)} \leq\left(\frac{|z|}{\operatorname{Re} z}\right)^{N_{|1 / p-1 / 2|}} \quad(\operatorname{Re} z>0)
$$


By Proposition 1.2, the assertion will be proved provided we can show that a multiple of the above upper bound also serves a slower bound for $\left\|G_{p}(z)\right\|_{\mathcal{L}\left(L^{p}\right)}$. This will be carried out in the following lemma.

Lemma 2.2. Let $1 \leq p \leq \infty$. Then

$$
\left\|G_{p}(z)\right\|_{\mathcal{L}\left(L^{p}\right)} \geq 2^{\frac{-N}{2 p}}\left(\frac{|z|}{\operatorname{Re} z}\right)^{N_{|1 / p-1 / 2|}} \quad(\operatorname{Re} z>0) .
$$

Proof. As we said before and by taking into account (2.1), it suffices to consider the case where $1<p \leq 2$. Fix $z \in \Sigma_{\pi / 2}$ and consider the function $f: \mathbb{R}^{N} \rightarrow \mathbb{C}$ defined by

$$
f(x):=\exp \left(\frac{-|x|^{2}}{\bar{z}}\right) .
$$

Choosing $q$ such that $\frac{1}{p}+\frac{1}{q}=1$ we verify that

$$
\|f\|_{L^{q}}=\left(\frac{\pi}{q}\right)^{\frac{N}{2 q}}\left(\frac{|z|^{2}}{\operatorname{Re} z}\right)^{\frac{N}{2 q}} .
$$

Let $x, y \in \mathbb{R}^{N}$ and recall that $z \in \Sigma_{\pi / 2}$. Then

$$
\begin{aligned}
-\frac{|x-y|^{2}}{z}-\frac{|x|^{2}}{\bar{z}}= & -2 \operatorname{Re}\left(\frac{1}{z}\right)\left|x-\frac{y}{2}\right|^{2}+2 i x y \operatorname{Im}\left(\frac{1}{z}\right) \\
& -|y|^{2}\left(\frac{1}{z}-\frac{1}{2} \operatorname{Re}\left(\frac{1}{z}\right)\right) .
\end{aligned}
$$

Moreover,

$$
\begin{aligned}
G_{q}\left(\frac{z}{4}\right) f(y)= & \left(\frac{1}{\pi z}\right)^{N / 2} \int_{\mathbb{R}^{n}} \exp \left(-\frac{|x-y|^{2}}{z}-\frac{|x|^{2}}{\bar{z}}\right) d x \\
= & \left(\frac{1}{\pi z}\right)^{N / 2} \int_{\mathbb{R}^{n}} \exp \left[-2|x|^{2} \operatorname{Re}\left(\frac{1}{z}\right)+2 i x y \operatorname{Im}\left(\frac{1}{z}\right)\right] d x \\
& \times \exp \left[-\frac{|y|^{2}}{2} \operatorname{Re}\left(\frac{1}{z}\right)\right] \\
= & \left(\frac{1}{\pi z}\right)^{N / 2}\left(\frac{1}{4 \operatorname{Re}\left(\frac{1}{z}\right)}\right)^{N / 2} \\
& \times \int_{\mathbb{R}^{n}} \exp \left[\frac{-|x|^{2}}{2}\right] \exp \left[i x y \frac{\operatorname{Im}\left(z^{-1}\right)}{\left(\operatorname{Re}\left(z^{-1}\right)\right)^{1 / 2}}\right] d x \exp \left[-\frac{|y|^{2}}{2} \operatorname{Re}\left(\frac{1}{z}\right)\right] \\
= & \left(\frac{1}{\pi z}\right)^{N / 2}\left(\frac{1}{4 \operatorname{Re}\left(\frac{1}{z}\right)}\right)^{N / 2}(2 \pi)^{N / 2} \\
& \times \exp \left[\frac{-|y|^{2}}{2} \frac{\left(\operatorname{Im}\left(z^{-1}\right)\right)^{2}}{\operatorname{Re}\left(z^{-1}\right)}\right] \exp \left[-\frac{|y|^{2}}{2} \operatorname{Re}\left(\frac{1}{z}\right)\right]
\end{aligned}
$$

where in the last step we used the fact that the function $x \mapsto \exp \left(\frac{-|x|^{2}}{2}\right)$ is a fixed point of the Fourier transform. Furthermore,

$$
\left|G_{q}\left(\frac{z}{4}\right) f(y)\right|=\left(\frac{|z|}{2 \operatorname{Re} z}\right)^{N / 2} \exp \left[-\frac{|y|^{2}}{2 \operatorname{Re} z}\right] \quad\left(y \in \mathbb{R}^{N}\right)
$$


and since $\int_{\mathbb{R}^{N}} \exp \left(\frac{-|x|^{2}}{2}\right) d x=(2 \pi)^{N / 2}$, we obtain

$$
\left\|G_{q}\left(\frac{z}{4}\right) f\right\|_{L^{q}}=\left(\frac{|z|}{2 \operatorname{Re} z}\right)^{N / 2}\left(\frac{2 \pi}{q}\right)^{\frac{N}{2 q}}(\operatorname{Re} z)^{\frac{N}{2 q}} .
$$

Combining this equality with (2.4) we see that

$$
\left\|G_{q}\left(\frac{z}{4}\right)\right\|_{\mathcal{L}\left(L^{q}\right)} \geq \frac{\left\|G_{q}\left(\frac{z}{4}\right) f\right\|_{L_{q}}}{\|f\|_{L^{q}}}=2^{\frac{-N}{2 p}}\left(\frac{|z|}{\operatorname{Re} z}\right)^{N(1 / p-1 / 2)} .
$$

Finally, since $\left\|G_{q}(z)\right\|_{\mathcal{L}\left(L^{q}\right)}=\left\|G_{p}(z)\right\|_{\mathcal{L}\left(L^{p}\right)}$ for $\frac{1}{p}+\frac{1}{q}=1$, it follows that

$$
\left\|G_{p}(z)\right\|_{\mathcal{L}\left(L^{p}\right)} \geq 2^{\frac{-N}{2 p}}\left(\frac{|z|}{\operatorname{Re} z}\right)^{N_{|1 / p-1 / 2|}} \quad(\operatorname{Re} z>0) .
$$

Remark 2.3 (Integrated semigroups). Boyadzhiev and de Laubenfels [B-deL] were the first to show that norm-estimates of holomorphic semigroups with generator $A$ on the right halfplane are equivalent to the fact that $i A$ generates a $k$-times integrated semigroup for suitable $k \in \mathbb{N}$ (or, what is the same, a $(w-A)^{-k_{-}}$ regularized semigroup; see [deL], [Ar] and [Hi1] for the definition and properties of these objects). This technique has been considerably refined in [El1]. Thus the estimate (2.2) tells us that $i \Delta$ generates an $\alpha$-times integrated semigroup provided $\alpha>N\left|\frac{1}{2}-\frac{1}{p}\right|$. See [B-deL] and [deL, Theorem 10.1] for entire $\alpha$ and [El1, Chapter VI.A] for non-entire $\alpha$. Observe that (2.3) also gives lower bounds: in fact, if $\alpha<N\left|\frac{1}{2}-\frac{1}{p}\right|$, then $i \Delta$ does not generate an $\alpha$-times integrated semigroup on $L^{p}\left(\mathbb{R}^{N}\right)$. For $\alpha \in \mathbb{N}$ this follows from [B-deL], [deL, Theorem 10.3] and for noninteger $\alpha$ from [El1, Chapter VI.A].

Former results of this kind were proved by Fourier multiplier theory (see [Hi1] and [Hi2] also for more general types of operators). By that method it is also possible to get precise information about the critical value $\alpha_{0}=N\left|\frac{1}{2}-\frac{1}{p}\right|$ : if $1<p<\infty$, then $i \Delta$ generates an $\alpha_{0}$-times integrated semigroup on $L^{p}\left(\mathbb{R}^{N}\right)$; however, if $p=1$, then $i \Delta$ does not generate an $\alpha_{0}$-times integrated semigroup on $L^{1}\left(\mathbb{R}^{N}\right)$ (see [Hi1]). So far it seems not possible to prove these statements by the holomorphy method.

\section{Bounded IMAGinARY POWERS AND THE RiEMANn-LiOUVILlE SEMigroup}

Interesting examples of boundary values of holomorphic semigroups occur in connection with fractional powers of operators. In fact, initiated by the regularity results due to Dore and Venni, much attention was recently given to operators having bounded imaginary powers. For detailed information on this subject we refer to $[\mathrm{Am}],[\mathrm{D}-\mathrm{V}],[\mathrm{Pr}],[\mathrm{P}-\mathrm{S}]$ and the references given there. If $A$ is sectorial and invertible, this actually means by definition that the holomorphic semigroup $\left(A^{-z}\right)_{\operatorname{Re}} z>0$ has a boundary value. We want to make this more precise.

Let $A$ be a densely defined operator in a Banach space $E$ such that there exist constants $M>0, \varphi \in(0, \pi)$ such that

$$
\sigma(A) \subset \Sigma_{\varphi} \quad \text { and } \quad\|R(\lambda, A)\| \leq \frac{M}{1+|\lambda|} \quad \text { for all } \lambda \in \mathbb{C} \backslash \overline{\Sigma_{\varphi}}
$$

Then one defines the fractional powers $A^{-z}$ of $A$ by

$$
A^{-z}:=\frac{1}{2 \pi i} \int_{C} \lambda^{-z}(A-\lambda)^{-1} d \lambda, \quad \operatorname{Re} z>0,
$$


where the path $C$ runs in $\rho(A)$ from $\infty e^{-i \varphi}$ to $\infty e^{i \varphi}, w<\varphi<\pi$, avoiding $\mathbb{R}_{\text {- }}$ and the origin (cf. [Kom]). It is well-known that the family $\left(A^{-z}\right)_{\operatorname{Re}} z>0$ is a holomorphic $C_{0}$-semigroup on $E$ of angle $\pi / 2$ (cf. [Am, Theorem 4.6.2]). In the case where $-A$ generates an exponentially stable $C_{0}$-semigroup $T$, the operator $A^{-z}$ is also given by the formula

$$
A^{-z} x=\frac{1}{\Gamma(z)} \int_{0}^{\infty} t^{z-1} T(t) x d t, \quad \operatorname{Re} z>0,
$$

for all $x \in E$ (cf. [Pa, p. 70]). We say that $A$ admits bounded imaginary powers if

$$
\sup _{z \in D}\left\|A^{-z}\right\|<\infty .
$$

By Proposition 1.1 and the subsequent remarks, condition (3.3) implies that $\left(A^{-z}\right)_{\operatorname{Re}} z>0$ has a boundary group which is denoted by $\left(A^{-i s}\right)_{s \in \mathbb{R}}$.

As an example we consider the operator $A$ in $L^{p}(0,1)$ given by

$$
D(A)=\left\{f \in W^{1, p}(0,1) ; f(0)=0\right\} \quad \text { and } \quad A f=f^{\prime} .
$$

Note that the operator $-A$ generates the semigroup $T$ on $L^{p}(0,1), 1 \leq p<\infty$, given by

$$
(T(t) f)(x)= \begin{cases}f(x-t) & \text { if } t \leq x \\ 0 & \text { if } t>x\end{cases}
$$

Furthermore, the semigroup $T$ is positive and contractive for all $p \in[1, \infty)$. Thus, in order to prove the boundedness of the imaginary powers of $A$, we may use the transference principle due to Coifman and Weiss $[\mathrm{C}-\mathrm{W}]$. By this we mean the following result (cf. [Am, Example 4.7.3c]):

Let $1<p<\infty,(\Omega, \mu)$ be a $\sigma$-finite measure space and $-A$ be the generator of a positive contraction semigroup on $L^{p}(\Omega, \mu)$. Then there exists a constant $C_{p}$ (depending only on $p$ ) such that

$$
\left\|A^{i s}\right\|_{\mathcal{L}\left(L^{p}(\Omega)\right)} \leq C_{p}\left(1+s^{2}\right) e^{|s| \frac{\pi}{2}} \quad(s \in \mathbb{R}) .
$$

Applying this result to the operator $A$ defined in (3.4), we see that $A$ has bounded imaginary powers whenever $1<p<\infty$ and that $A^{-i s}$ satisfies an estimate of the form

$$
\left\|A^{-i s}\right\|_{\mathcal{L}\left(L^{p}(0,1)\right)} \leq C_{p}\left(1+s^{2}\right) e^{\frac{\pi}{2}|s|} \quad(s \in \mathbb{R}),
$$

where the constant $C_{p}$ depends only on $p \in(1, \infty)$. Since $T(t)=0$ for $t \geq 1$, formula (3.2) is valid and we obtain

$$
\left(A^{-z} f\right)(x)=\frac{1}{\Gamma(z)} \int_{0}^{x}(x-y)^{z-1} f(y) d y, \quad x \in(0,1),
$$

i.e. $\left(A^{-z}\right)_{\operatorname{Re} z>0}$ is the Riemann-Liouville semigroup (see [H-P, Section 23.16]). Thus we proved that the Riemann-Liouville semigroup has a boundary group if $1<p<\infty$. This extends [H-P, p. 665] where the corresponding result is proved for $p=2$. 
The situation is different for $p=1$. Since $A^{-z}$ is an integral operator, the norm of $A^{-z}$ is given by

$$
\begin{aligned}
\left\|A^{-z}\right\|_{\mathcal{L}\left(L^{1}(0,1)\right)} & =\sup _{y \in[0,1]} \frac{1}{|\Gamma(z)|} \int_{y}^{1}\left|(x-y)^{z-1}\right| d x \\
& =\frac{1}{|\Gamma(z)|} \sup _{y \in[0,1]} \int_{y}^{1}(x-y)^{\operatorname{Re} z-1} d x=\frac{1}{|\Gamma(z)|} \frac{1}{\operatorname{Re} z} .
\end{aligned}
$$

Thus $\left(A^{-z}\right)_{\operatorname{Re}} z>0$ does not have a boundary group if $p=1$.

Summing up, we obtain the following result (which is proved in [H-P, Section 23.16] for $p=2$ ). Denote by $J$ the Riemann-Liouville semigroup on $L^{p}(0,1)$, $1 \leq p<\infty$, i.e. $J$ is given by

$$
J(z) f(x):=\frac{1}{\Gamma(z)} \int_{0}^{x}(x-y)^{z-1} f(y) d y \quad(\operatorname{Re} z>0) .
$$

Denote by $G$ the generator of $J$.

Theorem 3.1. For $1<p<\infty$, the operator $i G$ generates a $C_{0}$-group $\left(e^{i G s}\right)_{s \in \mathbb{R}}$ on $L^{p}(0,1)$ which satisfies

$$
\left\|e^{i G s}\right\|_{\mathcal{L}\left(L^{p}(j 0,1)\right)} \leq C_{p}\left(1+s^{2}\right) e^{|s| \frac{\pi}{2}} \quad(s \in \mathbb{R})
$$

where the constant $C_{p}$ depends only on $p$. If $p=1$, then $i G$ does not generate a semigroup.

We remark that $\sigma(G)=\varnothing$ since $J$ is nilpotent and that $G$ has compact resolvent since $J(t)$ is compact for all $t>0$.

Remark 3.2 (the vector-valued case). An alternative way to prove (3.5) is to use Fourier multiplier theory (see $[\mathrm{D}-\mathrm{V}$, Theorem 3.1$]$ ). This approach works also in the vector-valued case. More precisely, let $1<p<\infty$ and let $E:=L^{p}((0,1), X)$, where $X$ is a Banach space satisfying the UMD-property. Then Dore and Venni $[\mathrm{D}-\mathrm{V}$, Theorem 3.1] showed that the estimate (3.5) holds. Define $J$ on $E$ by (3.7). Then $J$ is a holomorphic semigroup on $E$ of angle $\frac{\pi}{2}$. We deduce from Proposition 1.2 that $J$ has a boundary group.

\section{Characterization of holomorphic semigroups BY THEIR BOUNDARY VALUES}

We start this section by proving the following converse result of Proposition 1.1.

Theorem 4.1. Let $A$ be an operator in $E$ and let $\varphi \in(0, \pi / 2)$. Assume that $e^{ \pm i \varphi} A$ generate bounded $C_{0}$-semigroups on $E$. Then $A$ generates a bounded holomorphic semigroup of angle $\varphi$.

Our proof of Theorem 4.1 is based on the Hille-Yosida theorem and the following version of the Phragmen-Lindelöf theorem (cf. [Co, Corollary 6.4.4]).

Theorem 4.2 (Phragmen-Lindelöf). Let $\varphi \in(0, \pi / 2]$ and let $h: \overline{\Sigma_{\varphi}} \rightarrow E$ be continuous and holomorphic in $\Sigma_{\varphi}$. Let $\alpha=\frac{\pi}{2 \varphi}$. Assume that for all $\varepsilon>0$ there exists a constant $C>0$ such that

$$
\|h(z)\| \leq C e^{\varepsilon|z|^{\alpha}} \quad\left(z \in \Sigma_{\varphi}\right) .
$$

If $\left\|h\left(r e^{ \pm i \varphi}\right)\right\| \leq M$ for all $r>0$, then $\|h(z)\| \leq M$ for all $z \in \Sigma_{\varphi}$. 
Proof of Theorem 4.1. Denote by $T_{+}$and $T_{-}$the semigroups generated by $A_{+}:=$ $e^{i \varphi} A$ and $A_{-}:=e^{-i \varphi} A$, respectively. Let $M \geq 0$ such that $\left\|T_{ \pm}(t)\right\| \leq M$ for $t \geq 0$. Then

$$
\left\|R\left(\lambda, A_{ \pm}\right)\right\|=\left\|\int_{0}^{\infty} e^{-\lambda t} T_{ \pm}(t) d t\right\| \leq \frac{M}{\operatorname{Re} \lambda} \quad(\operatorname{Re} \lambda>0) .
$$

Hence if $\lambda \in \Sigma_{\varphi}^{+}$, one has

$$
\|R(\lambda, A)\|=\left\|R\left(\lambda, e^{i \varphi} A_{-}\right)\right\|=\left\|R\left(\lambda e^{-i \varphi}, A_{-}\right)\right\| \leq \frac{M}{\operatorname{Re}\left(\lambda e^{-i \varphi}\right)} \leq \frac{M}{|\lambda| \cos \varphi} .
$$

Similarly, $\|R(\lambda, A)\| \leq \frac{M}{|\lambda| \cos \varphi}$ if $\lambda \in \Sigma_{\varphi}^{-}$. We have shown that

$$
\|R(1, z A)\|=\left\|z^{-1} R\left(\frac{1}{z}, A\right)\right\| \leq \frac{M}{\cos \varphi} \quad\left(z \in \Sigma_{\varphi}\right) .
$$

Let $h_{n}(z):=\left(I-\frac{z}{n} A\right)^{-n}$, where $z \in \overline{\Sigma_{\varphi}}$ and $n \in \mathbb{N}$. Then $\left\|h_{n}(z)\right\| \leq M$ for $z=r e^{ \pm i \varphi}$ and

$$
\left\|h_{n}(z)\right\| \leq\left(\frac{M}{\cos \varphi}\right)^{n} \quad \text { for } z=r e^{ \pm i \alpha}, r \geq 0,|\alpha|<\varphi .
$$

It follows from the Phragmen-Lindelöf principle that $\left\|h_{n}(z)\right\| \leq M$ for all $z \in \overline{\Sigma_{\varphi}}$ and all $n \in \mathbb{N}$. Now we apply the Hille-Yosida theorem to conclude that $A$ generates a $C_{0}$-semigroup $T$. Since $\lim _{n \rightarrow \infty} h_{n}(t)=T(t)$ strongly $(t \geq 0)$, it follows from Vitali's theorem [H-P, Theorem 3.14.1] that $T$ has a holomorphic extension $\widetilde{T}$ to $\Sigma_{\varphi}$ satisfying $\|\widetilde{T}(z)\| \leq M$ for all $z \in \Sigma_{\varphi}$.

We now want to use Theorem 4.1 to give a new proof of the following classical characterization theorem for holomorphic semigroups.

Theorem 4.3. Let $A$ be an operator in $E$ and let $\varphi \in(0, \pi / 2)$. Then the following assertions are equivalent.

(i) The operator $A$ generates a bounded $C_{0}$-semigroup $T$ which has a bounded holomorphic extension to every sector $\Sigma_{\alpha}$ with $\alpha \in(0, \varphi)$.

(ii) The operator $A$ is densely defined, $\Sigma_{\varphi+\frac{\pi}{2}} \subset \rho(A)$ and

$$
\sup _{\lambda \in \Sigma_{\varphi+\pi / 2-\varepsilon}}\|\lambda R(\lambda, A)\|<\infty \quad \text { for all } \varepsilon>0 .
$$

The key to our proof is the following lemma due to Prüss, which allows us to deduce from the complex estimate (4.1) the Hille-Yosida condition on the positive real line. We refer to [Pr, Proposition 0.1] for the short proof.

Lemma 4.4 (Prüss). Let $h: \Sigma_{\pi / 2} \rightarrow E$ be holomorphic and $M \geq 0$ such that

$$
\begin{aligned}
\|\lambda h(\lambda)\| & \leq M, \\
\left\|\lambda^{2} h^{\prime}(\lambda)\right\| & \leq M
\end{aligned}
$$

for all $\lambda \in \Sigma_{\frac{\pi}{2}}$. Then

$$
\left\|r^{n+1} h^{(n)}(r) / n !\right\| \leq 2 M \quad(r>0, n \in \mathbb{N}) .
$$


Proof of Theorem 4.3. (ii) $\Rightarrow$ (i): Let $M \geq 1$ such that $M \geq \sup _{\lambda} \in \Sigma_{\pi / 2}\|\lambda R(\lambda, A)\|$ $<\infty$. Note that $(-1)^{n} R(\lambda, A)^{(n)} / n !=R(\lambda, A)^{n+1}$. In particular, $\left\|\lambda^{2} R(\lambda, A)^{\prime}\right\| \leq$ $M^{2}(\operatorname{Re} \lambda>0)$. It follows from Lemma 4.4 that

$$
\left\|(r R(r, A))^{n+1}\right\|=\left\|r^{n+1} R(r, A)^{(n)} / n !\right\| \leq 2 M^{2} \quad(r>0) .
$$

By the Hille-Yosida theorem this implies that $A$ generates a bounded $C_{0}$-semigroup. In this argument we can replace $A$ by $e^{ \pm i \alpha} A$ for all $\alpha \in(0, \varphi)$. Now Theorem 4.1 implies (i).

(i) $\Rightarrow$ (ii): This is contained in the proof of Theorem 4.1.

If $T$ is a holomorphic semigroup of angle $\varphi$ which admits a boundary group and $z \in \Sigma_{\varphi}$, then $\|T(z)\|$ can be estimated by the norm of the boundary group. More specifically, the following holds true. Denote by $s(A):=\sup \{\operatorname{Re} \lambda ; \lambda \in \sigma(A)\}$ the spectral bound of $A$. Note that assertion (i) of the following proposition follows from the proof of Theorem 4.1. Here we give a direct argument.

Proposition 4.5. Let $A$ be the generator of a holomorphic semigroup $T$ of angle $\varphi \in(0, \pi / 2]$ satisfying (1.4). Assume that

$$
\left\|T\left(r e^{ \pm i \varphi}\right)\right\| \leq M \quad(r \geq 0) .
$$

Then the following hold.

(i) If $\varphi<\pi / 2$, then $\|T(z)\| \leq M$ for all $z \in \Sigma_{\varphi}$.

(ii) If $\varphi=\pi / 2$, then $\|T(z)\| \leq M e^{s(A) \operatorname{Re} z}$ for all $z \in \Sigma_{\pi / 2}$.

Proof. (i) Assume that $\varphi<\pi / 2$. Let $z \in \sigma_{\varphi}$. Then there exist $s, t \geq 0$ such that $z=t e^{i \varphi}+s e^{-i \varphi}$. Thus by (1.5), $T(z)=T\left(t e^{i \varphi}+s e^{-i \varphi}\right)$. Hence $\|T(z)\| \leq M^{2}$ for all $z \in \Sigma_{\varphi}$. It follows from Theorem 4.2 that $\|T(z)\| \leq M$ for all $z \in \overline{\Sigma_{\varphi}}$.

(ii) Assume that $\varphi=\pi / 2$. Replacing $A$ by $A-s(A)$ if necessary, we may assume that $s(A)=0$. Let $\varepsilon>0$. Since $s(A)=\omega(A)$ (cf. [Na, Theorem A-III, 6.6]), there exists $C>0$ such that $\|T(t)\| \leq C e^{\varepsilon t}$ for all $t \geq 0$. Let $z=t+i r \in \Sigma_{\pi / 2}$. Then by $(1.3)$

$$
\|T(z)\|=\|T(i r) T(t)\| \leq M C e^{\varepsilon t} \leq M C e^{\varepsilon|z|} .
$$

It follows from Theorem 4.2 that $\|T(z)\| \leq M$ for all $z \in \Sigma_{\varphi}$.

The example of the semigroup $T(z)=e^{w z}, w \geq 0$, on $\mathbb{C}$ shows that the estimate in (ii) is optimal.

Corollary 4.6. Let $A$ be the generator of a holomorphic semigroup $T$ of angle $\varphi \in(0, \pi / 2)$ satisfying (1.4). Assume that

$$
\left\|T\left(r e^{ \pm i \varphi}\right)\right\| \leq M e^{w r} \quad(r \geq 0)
$$

where $M \geq 0$ and $w \in \mathbb{R}$. Then

$$
\|T(z)\| \leq M e^{\operatorname{Re} z \frac{w}{\cos \varphi}} \quad \text { for all } z \in \overline{\Sigma_{\varphi}} .
$$

Proof. Apply Proposition 4.5 to $A-\frac{w}{\cos \varphi}$.

For later purposes we add two further corollaries of Theorem 4.1.

Corollary 4.7. Let $\varphi \in(0, \pi / 2)$ and assume that $e^{ \pm i \varphi} A$ generate $C_{0}$-semigroups $T_{ \pm \varphi}$. Then A generates a holomorphic semigroup of angle $\varphi$ with boundary groups $T_{ \pm \varphi}$. 
Proof. Let $\left\|T_{ \pm \varphi}(t)\right\| \leq M e^{\omega t}(t \geq 0)$ for suitable constants $M, \omega \geq 0$. Set $\mu:=\frac{\omega}{\cos \varphi}$. Then $e^{ \pm i \varphi}(A-\mu)$ generate bounded $C_{0}$-semigroups. So by Theorem 4.1, $A-\mu$ generates a bounded holomorphic semigroup of angle $\varphi$.

Corollary 4.8. Assume that $A$ generates a $C_{0}$-semigroup $T$ and that $i A$ generates a $C_{0}$-group $U$. Then $T$ has a holomorphic extension of angle $\pi / 2$ and $U$ is the boundary group of $T$.

Proof. By Corollary 4.7, the operator $\exp \left( \pm i \frac{\pi}{4}\right) A$ generates a holomorphic semigroup. Thus $e^{i \theta} A$ generates a $C_{0}$-semigroup for all $\theta \in(-\varphi, \varphi)$. It follows from Corollary 4.7 again that $A$ generates a holomorphic semigroup of angle $\pi / 2$. By Proposition 1.1, $U$ is its boundary group.

Remark 4.9. Concerning Theorem 4.1, our point was to give a proof based on the Hille-Yosida theorem. We then deduced from Theorem 4.1 the characterization theorem for holomorphic semigroups (Theorem 4.3) with the help of Prüss' lemma. One can also go the other way around: If $e^{ \pm i \varphi} A$ generate bounded semigroups, where $\varphi \in(0, \pi / 2)$, one sees easily that $\|\lambda R(\lambda, A)\|$ is bounded on each sector $\Sigma_{\varphi+\pi / 2-\varepsilon}, \varepsilon>0$. Thus $A$ generates a holomorphic semigroup of angle $\varphi$. This is precisely the argument given by Kato [Ka, Theorem 9.1.23].

\section{Spectral characterization of boundary groups}

Let $A$ be an operator in $E$ and assume that $i A$ generates a $C_{0}$-group $U$ on $E$. In this section we look for spectral conditions on $A$ which imply that $A$ generates a holomorphic semigroup $T$ such that $U$ is the boundary value of $T$.

An obvious necessary condition for this is that the spectrum of $A$ is located in a left halfplane, i.e. $s(A)<\infty$. However, this condition is not sufficient, in general. In fact, consider the generator $B$ of the Riemann-Liouville semigroup on $L^{p}(0,1)$, $p \in(1, \infty)$, and let $A=-B$. Then $s(A)=-\infty$ and $i A$ generates a group. But $A$ does not generate a semigroup. Nevertheless, the condition $s(A)<\infty$ is sufficient provided $U$ satisfies a certain growth condition.

Theorem 5.1. Let $A$ be an operator in $E$ such that $i A$ generates a $C_{0}$-semigroup $U$ on $E$. Assume that there exists a dense subspace $F$ of $E$ such that for all $x \in F$ there exist constants $C \geq 0$ and $k \in \mathbb{N}$ (depending on $x$ ) such that

$$
\|U(t) x\| \leq C(1+|t|)^{k}\|x\| \quad(t \in \mathbb{R}) .
$$

If $s(A)<\infty$, then $A$ generates a holomorphic $C_{0}$-semigroup on $E$ (whose boundary group is $U)$.

The key to our proof is the following result of Phragmen-Lindelöf type, whose proof is based on the idea to compensate the factor $1 / \sin \varphi$ by an appropriate function (cf. [Kor] and $[\mathrm{A}-\mathrm{B}]$ ).

Proposition 5.2. Let $r: \overline{\Sigma_{\pi / 2}} \rightarrow E$ be continuous and holomorphic in $\Sigma_{\pi / 2}$. Assume that there exist constants $C, M \geq 0, R_{0}>0, k \in \mathbb{N}$ such that

$$
\begin{aligned}
& \|r(\lambda)\| \leq \frac{C}{|\sin \varphi|^{k}} \quad\left(\operatorname{Re} \lambda \geq 0, \operatorname{Im} \lambda \neq 0,|\lambda| \geq R_{0}, \arg \lambda=\varphi\right) \text { and } \\
& \|r(i s)\| \leq M \quad(s \in \mathbb{R}) .
\end{aligned}
$$

Then $\|r(\lambda)\| \leq M$ for all $\lambda \in \mathbb{C}$ with $\operatorname{Re} \lambda \geq 0$. 
Proof. Choose $R \geq R_{0}$. For $k \in \mathbb{N}$ consider the holomorphic function $\lambda \mapsto$ $\left(1-\frac{\lambda^{2}}{R^{2}}\right)^{k} r(\lambda)$ on the domain $\{\lambda \in \mathbb{C} ;|\lambda|<R$, Re $\lambda>0\}$. Let $\lambda=R e^{i \varphi}$ for $\varphi \in(-\pi / 2, \pi / 2)$. If $\varphi \neq 0$, then

$$
\left\|\left(1-\frac{\lambda^{2}}{R^{2}}\right)^{k} r(\lambda)\right\|=\left|1-e^{i 2 \varphi}\right|^{k}\|r(\lambda)\|=2^{k}|\sin \varphi|^{k}\|r(\lambda)\| \leq 2^{k} C .
$$

If $\varphi=0$, then $\left\|\left(1-\frac{\lambda^{2}}{R^{2}}\right)^{k} r(\lambda)\right\|=0$. For $\lambda=i s, s \in[-R, R]$, one has

$$
\left\|\left(1-\frac{\lambda^{2}}{R^{2}}\right)^{k} r(\lambda)\right\|=\left(1+\frac{s^{2}}{R^{2}}\right)^{k}\|r(\lambda)\|<2^{k} M .
$$

It follows from the maximum principal that $\left\|\left(1-\frac{\lambda^{2}}{R^{2}}\right)^{k} r(\lambda)\right\| \leq 2^{k} \max \{C, M\}$ for all $\lambda \in \mathbb{C}$ satisfying $\operatorname{Re} \lambda \geq 0,|\lambda| \leq R$. Letting $R \rightarrow \infty$, one deduces that $\|r(\lambda)\| \leq 2^{k} \max \{C, M\}$ provided Re $\lambda \geq 0$. Now the Phragmen-Lendelöf principle implies that

$$
\|r(\lambda)\| \leq \sup _{s \in \mathbb{R}}\|r(i s)\| \leq M
$$

for all $\lambda \in \mathbb{C}$ with $\operatorname{Re} \lambda \geq 0$.

Proof of Theorem 5.1. Replacing $A$ by $A-w$ if necessary, we may assume that $s(A)<0$. Since $\pm i A$ generate a group on $E$, we have

$$
\sup _{\operatorname{Re} \lambda=0,|\lambda| \geq w}\|\lambda R(\lambda, A)\|<\infty \quad \text { for some } w \geq 0 .
$$

Since $s(A)<0$, it follows that $M:=\sup _{\operatorname{Re} \lambda=0}\|\lambda R(\lambda, A)\|<\infty$. Let $x \in F$. By hypothesis, there exist constants $k \in \mathbb{N}, C \geq 0$ such that $\|U(t) x\| \leq C(1+|t|)^{k}$ $(t \in \mathbb{R})$. Let $\lambda=r e^{i \varphi}$, where $r \geq 1$ and $\varphi \in(0, \pi / 2]$. Then

$$
\begin{aligned}
\|\lambda R(\lambda, A) x\| & =\left\|\lambda \int_{0}^{\infty} e^{i \lambda t} U(-t) d t\right\| \leq|\lambda| C \int_{0}^{\infty} e^{-|\operatorname{Im} \lambda| t}\left(1+t^{k}\right) d t \\
& \leq C|\lambda|\left(\frac{1}{|\operatorname{Im} \lambda|}+\frac{k !}{|\operatorname{Im} \lambda|^{k+1}}\right) \leq C(1+k !) \frac{1}{|\sin \varphi|^{k+1}} .
\end{aligned}
$$

Similarly, for $\lambda=r e^{i \varphi}$, where $r \geq 1$ and $\varphi \in[-\pi / 2,0)$, we have

$$
\|\lambda R(\lambda, A) x\| \leq C(1+k !) \frac{1}{|\sin \varphi|^{k+1}} .
$$

It follows from Proposition 5.2 that

$$
\|\lambda R(\lambda, A) x\| \leq M\|x\| \quad(\operatorname{Re} \lambda \geq 0) .
$$

Since $F$ is dense in $E$, we conclude that

$$
\|\lambda R(\lambda, A)\| \leq M \quad(\operatorname{Re} \lambda \geq 0) .
$$

Hence $A$ generates a holomorphic $C_{0}$-semigroup. Finally, Corollary 4.8 implies that $A$ generates a holomorphic semigroup $C_{0}$-semigroup of angle $\pi / 2$.

In the case where $U$ is a bounded group, Theorem 5.1 is due to Jazar [Ja1], [Ja2], who uses spectral calculus for the proof. A slightly more general version of Theorem 5.1 is given in [El2] with a very different and more complicated proof. 


\section{MiscellaneOUs REMARKS}

In this final section we collect some further information on boundary values of holomorphic semigroups.

Remark 6.1 (Cauchy-Riemann differential equations). Let $A$ and $B$ be generators of $C_{0}$-semigroups $T$ and $S$, respectively, such that $T(t) S(s)=S(s) T(t)$ for all $s, t \geq 0$. Then $V(t+i s):=T(t) S(s)$ defines a strongly continuous mapping $V$ : $\Sigma_{\pi / 2} \rightarrow \mathcal{L}(E)$ such that $V\left(z+z^{\prime}\right)=V(z) V\left(z^{\prime}\right)$ for all $z, z^{\prime} \in \Sigma_{\pi / 2}$. In analogy to the Cauchy-Riemann differential equations, we obtain the following result.

Proposition 6.2. The semigroup $V$ is holomorphic in $\Sigma_{\pi / 2}$ if and only if $B=i A$.

Proof. Assume first that $V$ is holomorphic and denote by $G$ the generator of $V$. Let $z \in \Sigma_{\pi / 2}, y \in E$ and $x=T(z) y$. Then $x \in D(G)$ and

$$
G x=\lim _{z \rightarrow 0, x \in \Sigma_{\pi / 2}} \frac{V(z) x-x}{z}=\lim _{t \rightarrow 0} \frac{T(t) x-x}{t}=A x=\lim _{s \rightarrow 0} \frac{S(s) x-x}{i s} .
$$

Hence $x \in D(A) \cap D(B)$ and $G x=A x=-i B x$. Now assume that $y \in D(G)$. Then $T(z) y \rightarrow y$ and $G T(z) y \rightarrow G y$ as $z \rightarrow 0$. It follows from the closedness of $A$ and $B$ that $y \in D(A) \cap D(B)$ and that $G y=A y=-i B y$. We have shown that $G \subset A$ and $G \subset-i B$. Hence $G=A=-i B$.

Remark 6.3 (Reflection principle). Let $E$ be the complexification of a real Banach space $E_{\mathbb{R}}$ and let $A$ be the generator of a $C_{0}$-semigroup $T$ on $E$. Then the following holds.

Proposition 6.4. Assume that $T(t) E_{\mathbb{R}} \subset E_{\mathbb{R}}$ for all $t \geq 0$ and let $\varphi \in(0, \pi / 2]$. If $e^{i \varphi} A$ generates a $C_{0}$-semigroup on $E$, then so does $e^{-i \varphi} A$.

Proof. By assumption and Proposition 1.2, $T$ has a strongly continuous extension to $\Sigma_{\varphi}^{+}$which is holomorphic in the interior of $\sigma_{\varphi}^{+}$. Define $T$ on $\Sigma_{\varphi}^{-}$by

$$
T(z):=\overline{T(\bar{z})} \text {. }
$$

Then, by Schwarz's reflection principle (cf. [Co, Chapter 9.1]), one obtains a holomorphic function $T: \Sigma_{\varphi} \rightarrow \mathcal{L}(E)$. Now the claim follows from Proposition 1.1.

\section{REFERENCES}

[Am] H. Amann, Linear and Quasilinear Parabolic Problems, Birkhäuser-Verlag, Basel, 1995. MR 96g:34088

[Ar] W. Arendt, Vector-valued Laplace transforms and Cauchy problems, Israel J. Math. 59 (1987), 327-352. MR 89a:47064

[A-B] W. Arendt, C. J. K. Batty, Tauberian theorems and stability of one-parameter semigroups, Trans. Amer. Math. Soc. 306 (1988), 837-852. MR 89g:47053

[B-deL] K. Boyadzhiev, R. deLaubenfels, Boundary values of holomorphic semigroups, Proc. Amer. Math. Soc. 118 (1993), 113-118. MR 93f:47043

[C-W] R. R. Coifman, G. Weiss, Transference Methods in Analysis, Conf. Board Math. Sci., Reg. Conf. Series Math., 31, Amer. Math. Soc., Providence, R.I., 1977. MR 58:2020

[Co] J. B. Conway, Functions of One Complex Variable, Springer-Verlag, New York, Heidelberg, Berlin, 1973. MR 56:5843

[Da] E. B. Davies, One-parameter Semigroups, Academic Press, London, 1980. MR 82i:47060

[deL] R. deLaubenfels, Existence Families, Functional Calculi and Evolution Equations, Springer Lecture Notes in Math. 1570, Berlin, 1994. MR 96b:47047

[D-V] G. Dore, A. Venni, On the closedness of the sum of two closed operators, Math. Z. 196, (1987), 189-201. MR 88m:47072 
[El1] O. El Mennaoui, Trace des semigroupes holomorphes singuliers a l'origine et comportement asymptotique, Ph. D. Thesis, Besançon, (1992).

[El2] - Holomorphic continuations of $C_{0}$-semigroups on Banach spaces, Semigroup Forum 49 (1994), 89-97. MR 95a:47036

[Go] J. A. Goldstein, Semigroups of Linear Operators and Applications, Oxford University Press, New York, 1985. MR 87c:47056

[Hi1] M. Hieber, Integrated semigroups and differential operators on $L^{p}$ spaces, Math. Ann. 291 (1991), 1-16. MR 92g:47052

[Hi2] — L $^{p}$ spectra of pseudo-differential operators generating integrated semigroups, Trans. Amer. Math. Soc. 347 (1995), 4023-4035. MR 95m:47099

[H-P] E. Hille, R. S. Phillips, Functional Analysis and Semigroups, Amer. Math. Soc., Providence, R.I., 1957. MR 19:664d

[Hö] L. Hörmander, Estimates for translation invariant operators in $L^{p}$ spaces, Acta Math. 104 (1960), 93-140. MR 22:12389

[Ja1] M. Jazar, Sur la théorie de la distribution spectrale et applications aux problèmes de Cauchy, Ph. D. Thesis, Poitiers, (1991).

[Ja2] M. Jazar, Fractional powers of the momentum of a spectral distribution, Proc. Amer. Math. Soc. 123 (1995), 1805-1813. MR 95g:47059

[Ka] T. Kato, Perturbation Theory of Linear Operators, Springer-Verlag, Berlin, Heidelberg, New York, 1966. MR 34:3324

[Kom] H. Komatsu, Fractional powers of operators, Pacific J. Math. 9 (1966), 285-346. MR 34:1862

[Kor] J. Korevaar, On Newman's quick way to the prime number theorem, Math. Intelligencer 4 (1982), 108-115. MR 84b:10063

[Na] R. Nagel (ed.), One-parameter Semigroups of Positive Operators, Springer Lecture Notes in Math. 1184, Berlin, 1985.

[Pa] A. Pazy, Semigroups of Linear Operators and Applications to Partial Differential Equations, Springer, New York, 1983. MR 85g:47061

[Pr] J. Prüss, Evolutionary Integral Equations and Applications, Birkhäuser, Verlag, Basel, 1993. MR 94h: 45010

[P-S] J. Prüss, H. Sohr, On operators with bounded imaginary powers in Banach spaces, Math. Z. 203 (1990), 429-452. MR 91b:47030

Mathematik V, Universität Ulm, D-89069 Ulm, Germany

Equipe de Mathématiques, Faculté des Sciences, Université Ibnon Zohr, Agadir, MoROCCO

Mathematisches Institut I, Universität Karlsruhe, Englerstrasse 2, D-76128 KarLSRUHe, GERMANY 